\title{
Altruistische Reziprozität
}

\section{Empirische Evidenz aus sequenziellen Diktator- und Taking-Spielen bei 11- jährigen Kindern ${ }^{1}$}

\section{Von Joël Berger}

Zusammenfassung: Eine grundlegende Frage der Soziologie ist jene nach der Entstehung sozialer Ordnung. Obwohl die Emergenz von Kooperationssystemen unter rationalen Egoisten möglich ist, stützen jüngere experimentelle Befunde die klassische soziologische Sichtweise, dass eine internalisierte Reziprozitätsnorm einen Beitrag zur Aufrechterhaltung von Kooperationsbeziehungen leistet. Im sequenziellen Diktatorspiel erwidern Versuchspersonen Vorleistungen eines ersten Akteurs auch dann, wenn strategische Motive ausgeschlossen werden können. Der vorliegende Beitrag berichtet die Resultate einer experimentellen Studie, in welcher die These einer Reziprozitätsnorm einem härteren Test unterzogen wird. Einerseits werden die Versuche mit 11-jährigen Schulkindern durchgeführt. Andererseits werden zusätzlich zu den Diktatorspielen sequenzielle Taking-Spiele durchgeführt. Die meisten Kinder erwidern Vorleistungen des ersten Spielers selbst dann, wenn sie seine gesamte Belohnung nehmen könnten. Die Befunde stützen die These, dass altruistische Reziprozität einen Beitrag zur Stabilität sozialer Systeme leistet.

\section{Einleitung}

Der Begriff der Reziprozität - der Gegenseitigkeit - spielt in der sozialwissenschaftlichen Literatur seit langer Zeit eine zentrale Rolle, beispielsweise in den Arbeiten von Georg Simmel (1950) oder Bronislaw Malinowski (1926). Alvin Gouldner (1960) spezifizierte das Konzept in einem einflussreichen Aufsatz. Er geht davon aus, dass Reziprozität einerseits als gegenseitiger Tausch von Gütern existiert und andererseits als universelle internalisierte Norm, die er als zentral für die Aufrechterhaltung sozialer Ordnung sieht (Gouldner 1960: 161, 171ff).

Robert Axelrod (1984) zeigte mit seinen berühmten Simulationsstudien, dass soziale Ordnung prinzipiell ohne eine Norm der Reziprozität möglich ist - selbst dann, wenn man unterstellt, dass der Mensch ein rationaler Egoist ist. Allerdings kann nachgewiesen werden, dass sich Menschen auch unter Bedingungen reziprok verhalten, in denen ein reiner homo oeconomicus opportunistisch handeln würde - beispielsweise im sequenziellen Diktatorspiel. Spieler 1 kann freiwillig einen Anteil eines Gutes, den er vom Versuchsleiter erhalten hat, an Spieler 2 abgeben. Entgegen den Vorhersagen enger Rational Choice-Ansätze und der klassischen Spieltheorie erwidert die Mehrheit der zweiten Spieler die Vorleistung von Spieler 1 mit exakt demselben Betrag, auch unter anonymen Bedingungen und wenn nur einmal gespielt wird. Strategische Motive können dadurch ausgeschlossen werden (Ben-Ner et al. 2004: 342; Diekmann 2004: 498ff).

Die vorliegende Untersuchung verfolgt das Ziel, die Experimente von Andreas Diekmann sowie von Avner Ben-Ner et al. zu replizieren - allerdings unter zweifach härteren Bedingungen. Einerseits werden anstelle der Drosophila der experimentellen Sozialforschung - den Studentinnen und Studenten - die Versuche mit 11-jährigen Kindern durchgeführt. Andererseits werden neben sequenziellen Diktatorspielen zusätzlich sequenzielle Taking-Spiele durchgeführt: Der zweite Spieler kann nicht nur entscheiden, ob er eine Vorleistung erwidern möchte oder nicht - er kann dem ersten Spieler auch sämtliche Einheiten wegnehmen, wodurch der

1 Ich bedanke mich für Anregungen und kritische Hinweise bei Sonja Vogt und zwei anonymen Gutachtern bzw. Gutachterinnen. 
Anreiz, sich eigennützig zu verhalten, erhöht wird. Durch einen Vergleich der beiden Spiele kann zudem die Robustheit der Resultate gegenüber Kategorien- und Demand-Effekten kontrolliert werden (Bardsley 2008).

Als theoretische Grundlage dient das ERC-Modell (theory of equity, reciprocity, and competition) von Gary Bolton und Axel Ockenfels (2000), welches im Gegensatz zu Gouldners Abhandlungen den Vorteil mit sich bringt, präzise Hypothesen darüber zu generieren, wie sich die Versuchspersonen in den verschiedenen Treatment-Bedingungen verhalten werden.

Der Befund, dass bereits Grundschulkinder, die ihren Sozialisationsprozess noch nicht abgeschlossen haben, ihr Entscheidungsverhalten an einer Reziprozitätsnorm orientieren, wäre ein Hinweis für die tiefen Wurzeln der Reziprozität im Verhaltensrepertoire des Menschen (Fehr / Rockenbach 2008). Die These, dass eine Norm der Reziprozität einen wesentlichen Beitrag zur Stabilität sozialer Systeme leistet, würde dadurch gestützt.

Im nachfolgenden Teil werden zentrale Konzepte und Theorien der Reziprozität vorgestellt (2). Darauf wird auf die Entwicklung von prosozialen Verhaltensweisen bei Kindern eingegangen (3). Anschließend werden das sequenzielle Diktator- und das sequenzielle TakingSpiel eingeführt und die vier Treatment-Bedingungen vorgestellt um Hypothesen darüber zu generieren, welches Verhalten in den verschiedenen Experimentalbedingungen zu erwarten ist. Zudem wird der Ablauf des Experiments beschrieben (4). Nach den empirischen Analysen (5) werden die Befunde diskutiert, ein Fazit gezogen und ein Ausblick auf weiterführende Forschungsfragen gegeben (6).

\section{Konzepte, Theorien und Modelle der Reziprozität}

\subsection{Konzepte und Theorien der Reziprozität}

Thomas Hobbes vertrat bekanntlich die Ansicht, dass nur die individuelle Unterordnung unter einen Leviathan den Krieg aller gegen aller verhindern könne (Hobbes 1968: 186). Robert Axelrod (1984) hat mit seinen berühmten Simulationsstudien jedoch gezeigt, dass selbst wenn der Mensch ein rationaler Egoist wäre, Kooperation ohne Zentralgewalt entstehen und stabil bleiben kann: Nämlich dann, wenn der durch wiederholten Tausch zu erzielende Nutzen höher ist, als der Nutzen, der durch gelegentliche Ausbeutung anderer Akteure realisiert werden kann. Wer einmal ausgenutzt wurde, wird seine Tauschbeziehungen mit dem Ausbeuter abbrechen. Aufgrund des stetigen Wegfalls von Tauschpartnern wird der Ausbeuter über längere Zeit weniger Gewinne einfahren, als wenn er die Vorleistungen der anderen Akteure erwidert und dadurch regelmäßig einen erfolgreichen Tausch realisieren kann. Da rationale Akteure eine solche Entwicklung vorhersehen, werden sie - vorausgesetzt der durch stetige Kooperation zu erzielende Nutzen ist abzüglich einer Diskontrate tatsächlich höher - sämtliche Vorleistungen erwidern. Es entstehen strategisch motivierte reziproke Austauschbeziehungen, wie sie Gouldner (1960) beschrieben hat. Eine Vorbedingung dafür ist, dass ein Schatten der Zukunft besteht: Die Chance, wiederholt auf den gleichen Akteur zu treffen, muss hinreichen hoch sein (Axelrod 1984: 11ff).

Martin Nowak und Karl Sigmund (2005) liefern eine Erklärung und empirische Evidenz dafür, dass Kooperationssysteme selbst dann aufrechterhalten werden können, wenn die Chance, zweimal auf denselben Akteur zu treffen, gegen null geht. Wird ein Individuum beim Ausbeuten eines anderen beobachtet und verbreitet sich diese Information im Netzwerk, verlieren rationale Akteure das Interesse daran, mit dem Ausbeuter zu kooperieren. Durch Reputation kann somit auch indirekte Reziprozität erklärt werden: A verhält sich freundlich gegenüber $B$ und $B$ verhält sich freundlich gegenüber $C$. Würde $B$ hingegen $C$ ausbeuten, dann würde $\mathrm{D}$, der die Interaktion zwischen $\mathrm{B}$ und $\mathrm{C}$ beobachtet, sich unfreundlich gegenüber $\mathrm{B}$ verhalten und die Information, dass B kein vertrauenswürdiger Tauschpartner ist, streuen. In der Folge 
würden weitere Akteure ihr Interesse daran verlieren, mit B Tauschbeziehungen zu pflegen. Beim Vorliegen solcher Reputationseffekte ist Defektion ebenfalls keine rationale Strategie, wenn die durch wiederholte Kooperation zu erzielenden Gewinne höher sind. ReputationsMechanismen ermöglichen beispielsweise die Aufrechterhaltung von Handel auf InternetPlattformen wie eBay (Diekmann / Wyder 2002). Robb Willer (2009) zeigt experimentell einen weiteren Effekt der Reputation: Gruppenmitglieder, die in ein Kollektivgut investieren und beobachtet werden, erzielen nicht nur erhöhte Kooperationsbereitschaft bei anderen Mitgliedern, sondern auch einen Statusgewinn innerhalb der Gruppe (Willer 2009: 23 f, 26ff). Eigeninteressierte Individuen mit Präferenzen für Macht und Status erhalten dadurch ein weiteres Motiv dafür, sich kooperativ zu verhalten.

Neben solch strategischer, mit der Annahme des Menschen als egoistischen Nutzenmaximierer kompatibler Reziprozität, scheint auch eine Form der altruistischen Reziprozität zu existieren:

„Prosoziales Verhalten gegenüber Fremden in einmaligen Situationen ist ein Beleg für Altruismus, sofern Dritte nicht davon erfahren und wie bei anonymen Spenden keine Reputationseffekte beteiligt sind" (Diekmann 2009: 205; vgl. auch Fehr / Fischbacher 2003: 785).

Ein rationaler, eigeninteressierter Akteur würde beispielsweise, wenn er allein im Ausland unterwegs ist, in einem Restaurant auch dann kein Trinkgeld geben, wenn er mit der Bedienung zufrieden ist. Sowohl eine erneute Begegnung wie auch die Möglichkeit einer Rufschädigung sind praktisch ausgeschlossen. Es handelt sich bei der Vergabe von Trinkgeld in einer solchen Situation folglich um positive altruistische Reziprozität (Diekmann 2004: 491; Diekmann 2009: 210) - um eine Verhaltensorientierung an einer Reziprozitätsnorm, wie sie Gouldner (1960: 171) unterstellt. Ein experimentelles Beispiel für positive altruistische Reziprozität stellt die empirische Bestätigung der Effizienzlohntheorie dar: Wird der Lohn von Arbeitern in einem simulierten Arbeitsmarkt gesteigert, steigt ihre Produktivität (Fehr / Kirchsteiger / Riedl 1993).

Neben positiver existiert auch negative altruistische ${ }^{2}$ Reziprozität: Ein Beispiel dafür ist das häufig beobachtete Ablehnen von geringen Beträgen im Ultimatumspiel ${ }^{3}$ (Camerer 2006: 43). Individuen, die kleine Anteile ablehnen, bestrafen das als unfair erachtete Verhalten des Gegenübers unter eigenen Kosten (Fehr / Fischbacher 2003: 786). In Experimenten strafen Probanden unkooperative Akteure regelmäßig, wenn sie selber nicht zu Schaden gekommen sind - ein beträchtlicher Anteil aller Personen auch dann, wenn sie für die Bestrafung Kosten auf sich nehmen müssen (Fehr / Fischbacher 2004). Joseph Henrich et al. (2006) weisen solches costly third party punishment, mit einer gewissen kulturspezifischen Varianz, in einer Vielzahl von Gesellschaftsformen, von Stammesgesellschaften bis Großstädten, nach.

Sowohl positive wie auch negative altruistische Reziprozität tragen zur Stabilität von Kooperationssystemen bei. Während positive Reziprozität, die Erwiderung einer freundlichen Handlung mit Freundlichkeit, Anreize für Kooperation schafft, verhindert negative Reziprozität, dass eigennützige Akteure diese Freundlichkeit ausnutzen. Wird etwa Trittbrettfahren

2 Die Begriffskombination ,negativ“ und ,altruistisch“ mag auf den ersten Blick irritieren. Der Term des Altruismus dient hier zur Abgrenzung von strategischer Reziprozität.

3 Im Ultimatumspiel kann Spieler 1 einen bestimmten Betrag beliebig zwischen sich und Spieler 2 aufteilen. Allerdings erhalten beide ihren Anteil nur dann, wenn Spieler 2 die Aufteilung akzeptiert. Lehnt Spieler 2 ab, erhalten beide nichts. Wäre Spieler 2 ein rationaler Egoist, würde er jeden noch so kleinen positiven Betrag akzeptieren (Bohnet 2009, S. 653). Tatsächlich lehnt etwa die Hälfte der Probandinnen und Probanden Anteile um 20\% ab (Camerer 2003, S. 43). 
im Öffentliche-Güter-Spiel ${ }^{4}$ bestraft, ist solches Verhalten auch für Egoisten nicht mehr rational und das Kooperationssystem bleibt stabil. Wird das Öffentliche-Güter-Spiel ohne Strafoption gespielt, bricht die Kooperation hingegen zusammen: Akteure, die einer Reziprozitätsnorm folgen, werden angesichts mangelnder Alternativen auf die Ausbeutung reagieren, indem sie ebenfalls keine Investitionen in das öffentliche Gut mehr vornehmen (Fehr / Gintis 2007: 47ff).

Zusammenfassend kann festgehalten werden, dass sowohl experimentell, wie auch in der sozialen Wirklichkeit, zwei Formen der Reziprozität auftreten. Einerseits existiert der reziproke Tausch, der mit reinem Eigeninteresse erklärt werden kann. Andererseits wird eine Form von Reziprozität beobachtet, die nicht mit den Vorhersagen enger Rational-Choice-Ansätze und der klassischen Spieltheorie vereinbar ist. Um solche, altruistische, Reziprozität erklären zu können, muss die Annahme eingeführt werden, dass Individuen nicht nur über egoistische, sondern zusätzlich über altruistische Präferenzen verfügen, beziehungsweise eine internalisierte Reziprozitätsnorm befolgen. Um klare Vorhersagen über das erwartete Verhalten in den verschiedenen Treatment-Bedingungen generieren zu können, stützen sich die nachfolgenden Analysen auf ein formales Modell der Reziprozität.

\subsection{Das ERC-Modell}

Als Reaktion auf die empirisch beobachteten Abweichungen von den Vorhersagen klassischer Ansätze in verschiedensten Experimenten, sind eine große Zahl formaler Fairness- und Reziprozitäts-Modelle entwickelt worden (für einen knappen Überblick: Falk / Fehr / Fischbacher 2008: 288 f). Im Wesentlichen wird in den neuen Modellen davon ausgegangen, dass menschliches Verhalten sowohl durch Eigeninteresse als auch durch altruistische Motive - beziehungsweise die Befolgung sozialer Normen - motiviert ist. Etliche Modelle stellen somit eine Synthese aus den lange Zeit als antagonistisch betrachteten Konzepten des eigennützigen homo oeconomicus und des von sozialen Normen determinierten homo sociologicus (Parsons 1973) dar (Fehr / Gintis 2007: 44ff). Vorliegend wird Diekmann (2004) gefolgt und Reziprozität als Erwiderung der Handlung des Gegenübers definiert. Zudem wird Reziprozität analog zu Gouldner (1960: 175) als kontinuierliche Größe betrachtet. Die getauschten Güter müssen nicht exakt den gleichen Wert haben. Folglich kann reziprokes Verhalten durch Ungleichheitsaversion im Sinne von Gary Bolton und Axel Ockenfels (2000) sowie Ockenfels (1999) erklärt werden. Ihr ERC-Modell (theory of equity, reciprocity, and competition) ist axiomatisch aufgebaut. Es wird die Existenz einer Nutzenfunktion (motivation function) postuliert, welche die besondere Eigenschaft aufweist, dass Akteure nicht nur aus materiellen Gewinnen Nutzen erzielen. Ein Akteur erleidet einen subjektiven Nutzenverlust, wenn sein Anteil an einem Gut vom durchschnittlich ausbezahlten Anteil abweicht. Erbringt eine erste Person gegenüber einer zweiten eine Vorleistung, ist die erste Person gegenüber dieser zweiten schlechter gestellt. Da Person 2 durch die relative Besserstellung einen Nutzenverlust erfahren würde, wird sie die Vorleistung von Person 1 erwidern und sich somit reziprok verhalten (Bolton / Ockenfels 2000:

4 Im Öffentliche-Güter-Spiel erhalten alle n Mitspielenden einen Anfangsbetrag, den sie in jeder der k Runden entweder selber behalten oder in einen kollektiven Fonds investieren können, wobei das Geld im Fonds mit einem Faktor $\mathrm{F}$ (wobei $1<\mathrm{F}<\mathrm{n}$ ) multipliziert und zwischen allen Mitspielenden gleichmäßig aufgeteilt wird. Die höchste kollektive Auszahlung ergibt sich, wenn alle den gesamten Betrag einzahlen. Allerdings hat jede Person einen individuellen Anreiz, ihren Betrag selber zu behalten (NashGleichgewicht): Investieren außer Spieler A alle in den Fonds, erzielt Spieler A die höchste Auszahlung. $\mathrm{Da}$ alle rationalen, eigeninteressierten Akteure so denken, investiert gemäß klassischen Modellvorhersagen niemand in den Fonds und das (kollektive) Pareto-Optimum wird verfehlt (Diekmann 2009, S. $120 \mathrm{f})$. 
16 ff; Diekmann 2004: 462 f; Diekmann 2009: 211 f). ${ }^{5}$ Für die hier verwendeten Spiele wird von folgender, mit den Axiomen des ERC-Modells übereinstimmender, Nutzenfunktion ausgegangen (vgl. Bolton / Ockenfels 2000: 174):

$u_{\mathrm{i}}=\mathrm{a}_{\mathrm{i}} \mathrm{y}_{\mathrm{i}}-\mathrm{b}_{\mathrm{i}}\left(1 / 2-\sigma_{\mathrm{i}}\right)^{2}$

Dabei bezeichnet $u_{i}$ den Nutzen von Individuum i, der abhängig ist vom materiellen Gewinn $\mathrm{y}_{\mathrm{i}}$ sowie von der Abweichung der eigenen Auszahlung von einem als fair erachteten Punkt im Falle des spezifizierten Modells von der Gleichheitsnorm. Bei zwei Spielern ist dies je die Hälfte des Betrags $\left(\sigma_{\mathrm{i}}-1 / 2\right.$; wobei $\left.\sigma_{\mathrm{i}}=\left(\mathrm{y}_{\mathrm{i}} /\left[\mathrm{y}_{1}+\mathrm{y}_{2}\right]\right)\right)$. Da nicht alle Individuen dieselben Präferenzen bezüglich Eigennutz und Fairness haben, werden die zwei individuellen Gewichtungsparameter $\mathrm{a}_{\mathrm{i}}$ (Gewichtungsparameter für eigennützige Präferenzen) und $\mathrm{b}_{\mathrm{i}}$ (Gewichtungsparameter für Ungleichheitsaversion) eingeführt, wobei gilt: $a_{i} \geq 0$ und $b_{i}>0$. Mit dem Verhältnis von $a_{i} / b_{i}$ können verschiedene Typen von Spielern unterschieden werden. Mit steigendem Eigeninteresse strebt das Verhältnis $a_{i} / b_{i}$ gegen unendlich, mit steigender Ungleichheitsaversion gegen null. Je nachdem, wie das Verhältnis von $\mathrm{a}_{\mathrm{i}}$ und $\mathrm{b}_{\mathrm{i}}$ beschaffen ist, wird früher oder später ein Schwellenwert erreicht, bei dessen Überschreiten zusätzlicher materieller Gewinn aufgrund der Abweichung von der Fairnessnorm mehr Kosten verursacht als Nutzen stiftet. Jedes Individuum wird daher versucht sein, seine Nutzenfunktion durch einen Trade-off zwischen materiellem Gewinn und normorientiertem Handeln zu maximieren (Bolton / Ockenfels 2000: $171 \mathrm{ff})$.

\section{Reziprozität und prosoziales Verhalten bei Kindern}

Da in der vorliegenden Untersuchung geprüft wird, ob bei Kindern im Grundschulalter bereits altruistische Reziprozität beobachtet werden kann, soll kurz auf die Entwicklung prosozialer Verhaltensweisen bei Kindern eingegangen werden. In der Entwicklungspsychologie wird davon ausgegangen, dass als Voraussetzung für prosoziales Verhalten eine theory of mind (Premack / Woodruff 1978) entwickelt worden sein muss (Robinson 2008: 442ff; Kienbaum / Schuhrke 2010202 f; Takagishi et al. 2010: 131). Darunter wird die Fähigkeit zur Perspektivenübernahme verstanden; eine Vorstellung davon, dass abgesehen vom eigenen Ich andere Personen mit anderen Bedürfnissen existieren (Robinson 2008: 442; Takagishi et al. 2010: 131 f; vgl. Batson / Moran 1999). Jutta Kienbaum und Bettina Schuhrke (2010) kommen in einem Überblick über entwicklungspsychologische Studien zum Schluss, dass sich solche empathischen Fähigkeiten in der Regel zwischen dem dritten und fünften Altersjahr entwickeln (Kienbaum / Schuhrke 2010: 203; vgl. Robinson 2008: 442).

Bezüglich der Aufteilung von Gütern berichtet William Damon (1984), basierend auf Interviews mit Kindern, dass die ersten Fairnessvorstellungen von striktem Egalitarismus geprägt sind. Ab einem Alter von ca. 8 Jahren werden sukzessive situationsspezifische Aufteilungsethiken entwickelt. Beispielsweise werden neben Gleichheit zusätzlich Leistung oder Bedürftigkeit beachtet. Neuere Untersuchungen bestätigen Damons Befund weitgehend, zeigen aber, dass der kontextunabhängige Egalitarismus bereits mit etwa 5 Jahren schrittweise zu Ende geht (Kienbaum / Schuhrke 2009: 193ff).

Während in früheren Untersuchungen Kinder häufig interviewt und aufgrund solcher Gespräche auf ihre Moralvorstellungen geschlossen wurde (z.B. Damon 1984), sind in den letzten Jahren vermehrt Experimente mit Fokus auf das Fairnessverhalten von Kindern durchgeführt

5 Dies ist eine konsequentialistische Definition von Reziprozität. Zur Zeit wird eine Debatte darüber geführt, ob eine intentionalistische Definition Reziprozität besser fassen kann. Dabei geht es um die Frage, ob ein Empfänger eher die Gabe eines ,Spenders` oder die Intention, die er hinter der Gabe vermutet, erwidert (vgl. Xiao / Bicchieri 2008; Fehr et al. 2008). 
worden. So weisen Haruto Takagishi et al. (2010: 135) nach, dass Kinder im Vorschulalter, die bereits eine theory of mind entwickelt haben, im Ultimatumspiel höhere Angebote machen als Gleichaltrige, welche die Fähigkeit zur Perspektivenübernahme noch nicht erlangt haben. Keith Murningham und Michael Saxon (1998) untersuchen das Verhalten von Kindern und Jugendlichen verschiedener Altersgruppen im Ultimatumspiel - allerdings ohne reelle Auszahlung. Kinder im Alter von 9 und 12 Jahren bieten häufiger die Hälfte des Betrages an als College-Studierende und verhalten sich noch kaum strategisch. Bei der Süßigkeit ,M\&M's“ werden öfters faire Angebote gemacht als bei Geld. Gerade die 9-Jährigen teilen ,M\&M's“ häufig in zwei gleich große Anteile auf; sowohl sich selbst als auch die andere Person begünstigende Angebote werden abgelehnt. Fehr et al. (2008) zeigen, dass Kinder im Alter von 7 bis 8 Jahren die egalitäre Aufteilungen von Süßigkeiten (z.B.,Smarties` oder Gummibärchen) eigennützigem Verhalten vorziehen. Bei 3-4-Jährigen überwiegt hingegen noch Egoismus. Joyce Benenson et al. (2007) beobachten altruistisches Verhalten in Diktatorspielen, wobei der Anteil der abgegebenen Einheiten (Stickers) bei 6-Jährigen, und noch stärker bei 9-Jährigen, höher liegt als bei 4-Jährigen.

Zusammenfassend wird im Folgenden aufgrund der relativ einheitlichen entwicklungspsychologischen Befunde davon ausgegangen, dass Kinder im Alter von rund 11 Jahren eine theory of mind entwickelt haben. Wenn Reziprozität zu den zentralen menschlichen Verhaltensweisen zählt, müsste reziprokes Verhalten daher bei Kindern in diesem Alter bereits beobachtbar sein.

\section{Experimentelles Design, Versuchspersonen und Ablauf}

\subsection{Reziprozität messen: Sequenzielles Diktatorspiel und sequenzielles Taking-Spiel}

Im einfachen Diktatorspiel kann Spieler 1 einen beliebigen Anteil eines Gutes an Spieler 2 abgeben. Der zweite Spieler hat keine Möglichkeit, das Verhalten von Spieler 1 zu beeinflussen oder darauf zu reagieren. Das sequenzielle Diktatorspiel besteht aus zwei Runden. Nach der ersten Runde wird Spieler 2, nachdem er von Spieler 1 einen Anteil eines Gutes mit bekanntem Umfang erhalten hat, selber zum Diktator. Spieler 2 kann entscheiden, welchen Anteil eines Gutes desselben Umfangs er an Spieler 1 abgeben möchte. Da Spieler 1 nach der Vergabe seines Anteils keinen Einfluss auf die Entscheidung von Spieler 2 hat, die Entscheidungssituation anonym ist und das Spiel nur einmal gespielt wird, hat Spieler 2 keinen strategischen Anreiz, sich reziprok zu verhalten. Rationale Egoisten würden nichts abgeben. Das sequenzielle Diktatorspiel ist somit eine einfache und sparsame Methode um altruistische Reziprozität zu messen (Diekmann 2004: 494). Dabei wird die Komplexität der Welt auf die relevante Grundstruktur reduziert, welche aus theoretischer Sicht das zu erklärende Verhalten generiert (Bohnet 2009: 640; Bardsley et al. 2010). Als eine spezifische Instanz des Modells wären etwa die Schweizer Bauern zu nennen, die ihre Produkte, beispielsweise Schnittblumen oder Honig, neben den Feldern zum Verkauf anbieten, ohne selber anwesend zu sein. Es steht lediglich ein Behälter für die Zahlungen bereit. Auf den einsamen Landstrassen ist die Anonymität hoch, Reputationseffekte sind praktisch ausgeschlossen. Dass sich das Geschäft für die Bauern lohnt, ist wohl einer Reziprozitätsnorm zuzuschreiben.

Sowohl Diekmann (2004) als auch Ben-Ner et al. (2004) finden in experimentellen Studien mit dem sequenziellen Diktatorspiel robuste Reziprozitätseffekte. In beiden Untersuchungen gibt die relative Mehrheit der Spieler - in beiden Fällen Studierende - denselben Anteil an Spieler 1 zurück, den sie vorgängig erhalten haben.

Zusätzlich zu den beiden sequenziellen Diktatorspielen werden vorliegend zwei sequenzielle Taking-Spiele (vgl. zum einfachen Taking-Spiel List 2007; Bardsley 2008) durchgeführt. Das 
sequenzielle Taking-Spiel funktioniert wie das sequenzielle Diktatorspiel, beinhaltet jedoch die zusätzliche Option, dem anderen Spieler Einheiten zu nehmen. ${ }^{6}$

List (2007) und Bardsley (2008) berichten, dass im anonymen Diktatorspiel deutlich weniger gegeben wird, wenn eine Taking-Option eingeführt wird. Die Autoren nennen als mögliche Erklärungen Kategorien- und Demand-Effekte. Ein Kategorieneffekt (Parducci / Wedell 1986) tritt dann auf, wenn die Skala - etwa die Anzahl der Kategorien - die Entscheidung der Individuen beeinflusst. Weil im Taking-Spiel im Gegensatz zum Diktatorspiel das Geben von 0 Einheiten nicht mehr zu den Extrempunkten der Skala zählt, könnte das Abgeben von 0 Einheiten als weniger unfreundlich wahrgenommen werden (Bardsley 2008: 129). Ein DemandEffekt kann dadurch verursacht werden, dass Probandinnen und Probanden aufgrund von, versteckten Hinweisen' auf, angemessenes Verhalten “ im Experiment schließen (Ornes 1962). So hat eine Versuchsperson im Diktatorspiel die Wahl zwischen der passiven Option ,Nichtstun und der aktiven Option ,Geben'. Versuchspersonen könnten folgern, dass ,Geben“ das vom Versuchsleiter erwartete Verhalten ist (Bardsley 2008: 129). Existieren hingegen neben ,Nichtstun' die beiden Handlungsoptionen ,Geben' und ,Nehmen', sinkt die Gefahr eines solchen Effekts. Zusätzlich erhöht sich im Taking-Spiel der Gewinn, der durch eigennütziges Verhalten erzielt werden kann. Das Taking-Spiel ermöglicht somit einen härteren Test der Reziprozitäts-These.

Ein informativer Vergleich des Entscheidungsverhaltens kann nicht nur innerhalb, sondern auch zwischen den Spielen gezogen werden. Wenn wirklich eine Reziprozitätsnorm und nicht die Orientierung an einer vorgegebenen Skala für das Geben verantwortlich ist, dürfte im sequenziellen Taking-Spiel der Anteil an Personen in Position 2, die eine Vorleistung von Spieler 1 erwidern, nicht geringer sein als im sequenziellen Diktatorspiel (vgl. Bardsley 2008: 124).

\subsection{Experimentaldesign}

Im Folgenden werden die 4 Treatment-Bedingungen beschrieben und mittels ERC-Modell Hypothesen darüber generiert, welches Entscheidungsverhalten in den jeweiligen Versuchsbedingungen zu erwarten ist. Als Belohnung wurde anstelle von Geld Gummibärchen verwendet, die sich in einem Experiment von Fehr et al. (2008) als geeignetes Incentive erwiesen. Alle Versuchspersonen erhielten 8 Gummibärchen.

Für das Testen der theoretisch erwarteten Effekte ist nur das Verhalten der Spieler in der zweiten Position von Belang. Um die Stichprobe effizient zu nutzen, wurde deshalb ein Vorgehen analog zu Diekmann (2004) gewählt: Sämtliche Probandinnen und Probanden nehmen den Platz von Spieler 2 ein. Der erste Spieler ist fiktiv, seine ,Entscheidung ' wird durch den Versuchsleiter festgelegt. Dem zweiten Spieler wird jedoch mitgeteilt, dass es sich bei Spieler 1 ebenfalls um eine Versuchsperson handelt.

Im sequenziellen Diktatorspiel (8, 0) wird der Versuchsperson, welche die Rolle des zweiten Spielers einnimmt, mitgeteilt, dass Spieler 1 alle 8 Gummibärchen für sicht behält. Spieler 2 hat darauf die Möglichkeit, zwischen 0 und 8 seiner eigenen Gummibärchen an Spieler 1 abzugeben. Im sequenziellen Diktatorspiel $(5,3)$ erhält der zweite Spieler vom ersten hingegen 3 Gummibärchen. Der zweite Spieler kann nun seinem Partner zwischen 0 und 8 seiner eigenen Einheiten zukommen lassen.

Wie im Diktatorspiel $(8,0)$ erfährt die Versuchsperson im sequenziellen Taking-Spiel (8, $0)$, dass der erste Spieler alle 8 Einheiten behalten möchte. Der zweite Spieler verfügt wiederum

6 Im Falle der Schweizer Bauern wäre es also nicht nur möglich, den Honig ohne Bezahlung mitzunehmen, sondern zusätzlich, die Kasse zu plündern. 
über die Möglichkeit, zwischen 0 und 8 Einheiten seiner Belohnung an den ersten Spieler abzugeben. Zusätzlich besteht die Option, vom ersten Spieler bis zu 8 Gummibärchen zu nehmen. Im Extremfall erhält der zweite Spieler 16 Einheiten, während der erste leer ausgeht. Im sequenziellen Taking-Spiel $(5,3)$ erhält Spieler 2 vom ersten Spieler 3 Gummibärchen. Er kann nun bis zu 8 Einheiten seiner eigenen Belohnung an seinen Partner abgeben. Alternativ kann er bis zu 5 Einheiten des ersten Spielers einfordern (3 Einheiten von Spieler 1 sind bereits in seinem Besitz).

Tabelle 1: Versuchsbedingungen

\begin{tabular}{lll}
\hline & $\begin{array}{l}\text { erste Runde, } \\
\text { Spieler 1 entscheidet }\end{array}$ & $\begin{array}{l}\text { zweite Runde, } \\
\text { Spieler 2 entscheidet }\end{array}$ \\
\hline Sequenzielles Diktatorspiel $(8,0)$ & behält 8 Einheiten, gibt 0 & $\begin{array}{l}\text { Hat 8 Einheiten. } \\
\text { Kann bis zu 8 Einheiten ge- } \\
\text { ben. }\end{array}$ \\
Sequenzielles Diktatorspiel $(5,3)$ & behält 5 Einheiten, gibt 3 & $\begin{array}{l}\text { Hat 11 Einheiten. } \\
\text { Kann bis zu 8 Einheiten ge- } \\
\text { ben. }\end{array}$ \\
& behält 8 Einheiten, gibt 0 & $\begin{array}{l}\text { Hat 8 Einheiten. } \\
\text { Kann bis zu 8 Einheiten geben } \\
\text { oder bis zu 8 zusätzliche Ein- } \\
\text { heiten nehmen. }\end{array}$ \\
& & $\begin{array}{l}\text { Hat 11 Einheiten. } \\
\text { Kann bis zu 8 Einheiten geben } \\
\text { oder bis zu 5 zusätzliche Ein- } \\
\text { heiten nehmen. }\end{array}$ \\
\hline Sequenzielles Taking-Spiel $(5,3)$ & behält 5 Einheiten, gibt 3 & \\
& &
\end{tabular}

${ }^{*}$ Spieler 1 ist fiktiv, Spieler 2 ist ein/e Proband/in und weiß nicht, dass Spieler 1 fiktiv ist.

\subsection{Hypothesen}

Da Kinder am Ende der Grundschulzeit eine theory of mind entwickelt haben und hinreichend sozialisiert sein sollten um ihr Verhalten an einer Reziprozitätsnorm zu orientieren, wird im folgenden das ERC-Modell verwendet, um Hypothesen über das zu erwartende Verhalten in den vier Treatment-Bedingungen zu generieren.

Das ERC-Modell geht davon aus, dass Individuen mit völlig eigennützigen Präferenzen (das Verhältnis der Gewichtungsparameter für Eigennutz $a_{i}$ und Ungleichheitsaversion $b_{i}$ geht gegen unendlich) auch dann nichts abgeben, wenn der Mitspieler ihnen einen Anteil seiner Belohnung abgegeben hat. Probanden, die sich perfekt reziprok verhalten $\left(\mathrm{a}_{\mathrm{i}} / \mathrm{b}_{\mathrm{i}}\right.$ geht gegen null), geben genau denselben Anteil zurück, den sie erhalten haben. Zudem sind Mischformen zwischen den beiden Extremen zu erwarten. Das Modell sieht jedoch keinen unbedingten Altruismus vor. Das Geben von mehr als 3 Einheiten in den (5, 3)-Bedingung würde einerseits zu Verlust an materiellem Gewinn, andererseits zu Nutzenverlust aufgrund der Abweichung von der Gleichheitsnorm führen. Für die D(8, 0)-Bedingung macht das ERC-Modell daher dieselbe Prognose wie klassische Modelle: Die Akteure werden nichts abgeben.

Hypothese 1: In der Versuchsbedingung D $(5,3)$ ist die zentrale Tendenz der abgegebene

Einheiten höher als in der Versuchsbedingung $D(8,0)$.

Im Taking-Spiel verschiebt sich der Bezugspunkt egoistischen Handelns. Ein reiner homo oeconomicus würde zusätzlich zu seinen eigenen 8 Einheiten die ganze Belohung des ersten Spielers einfordern. Sollten die Versuchspersonen auch im Taking-Spiel die Vorleistung des ersten Akteurs erwidern, wäre dies ein umso stärkerer Hinweis für das Wirken einer Reziprozitätsnorm. 
Hypothese 2: In der Versuchsbedingung $T(5,3)$ ist die zentrale Tendenz der abgegebenen Einheiten höher als in der Versuchsbedingung $T(8,0)$.

Hypothesen 1 und 2 beziehen sich auf den Vergleich der Aufteilungen innerhalb der beiden Diktator- beziehungsweise innerhalb der beiden Taking-Spiele. Zusätzlich soll eine Hypothese darüber formuliert werden, wie sich die Entscheidungen zwischen den beiden $(5,3)$-Versuchsgruppen unterscheiden werden. Das ERC-Modell prognostiziert wiederum, dass das Verhalten von absolutem Eigennutz bis zu völliger Reziprozität streut. Während eigennützige Individuen die Taking-Option nutzen, würden dies Kinder, welche aufgrund der Vorleistung von Spieler 1 der Gleichverteilungsnorm folgen, nicht tun. Der arithmetische Mittelwert, der gerade bei geringen Fallzahlen empfindlich auf Änderungen an den Rändern der Verteilung reagiert, dürfte aufgrund der eigennützigen Akteure in beiden Taking-Bedingungen tiefer liegen. Allerdings darf bei Gültigkeit des ERC-Modells der relative Anteil der reziprok handelnden Probanden im Taking-Spiel nicht tiefer liegen, wenn tatsächlich die Orientierung an einer Verteilungsnorm - und nicht ein Demand- oder Kategorieneffekt - für das beobachtete Verhalten verantwortlich ist. Der relative Anteil an Akteuren, der das freundliche Verhalten des ersten Spielers erwidert, sollte sich daher zwischen den beiden $(5,3)$-Bedingungen nicht unterscheiden, wenn die Entscheidungen der zweiten Spieler durch die Orientierung an einem als fair erachteten Punkt motiviert ist (Nullhypothese zu Hypothese 3). Sind hingegen Demandoder Kategorieneffekte für das prosoziale Verhalten verantwortlich, müsste ein Unterschied in den Anteilen der Akteure mit Fairnesspräferenzen beobachtbar sein (Hypothese 3). ${ }^{7}$

Hypothese 3: Der relative Anteil der Personen, der Einheiten gibt, ist in der Versuchsbedingung $T(5,3)$ kleiner als in der Versuchsbedingung $D(5,3)$.

\subsection{Versuchspersonen und Ablauf}

Die Experimente wurden im Frühjahr 2010 mit 96 Kindern aus fünf Grundschulkassen der fünften Jahrgangsstufe in drei eher ruralen Gemeinden der Schweizer Kantone Aargau und Bern durchgeführt. ${ }^{8}$ Die Kinder waren im Mittel 11-Jährig (mit 9 und 13 Jahren als Extremwerten). Innerhalb jeder Schulklasse wurden die 4 Versuchsbedingungen zufällig auf die Kinder verteilt.

Die jeweilige Klassenlehrperson instruierte die Schülerinnen und Schüler nach einer standardisierten Vorgabe über den Ablauf des Versuchs. ${ }^{9}$ Die Aufteilung der Gummibärchen erfolgte anschließend selbständig durch die Kinder. Der Experimentator war während des ganzen Ablaufs nicht anwesend.

Die einführenden Informationen wurden allgemein gehalten, damit nicht auf die verschiedenen Experimentalbedingungen geschlossen werden konnte. So wurde den Schülerinnen und Schülern mitgeteilt, dass sich jeweils zwei Klassen gemeinsam an einem Versuch über das Entscheidungsverhalten von Kindern beteiligen. Je ein Schulkind der einen Klasse und ein Schulkind der anderen Klasse könnten miteinander Gummibärchen aufteilen. Alle Schülerinnen und Schüler der anderen Klasse aus einem anderen Kanton hätten je 8 Gummibärchen erhalten, weil sie bei diesem Versuch mitmachen. Die anderen Schulkinder hätten sich bereits entschieden, wie sie ihre Belohnungen aufteilen möchten. Nun erhalte jede Schülerin und jeder

7 Zwischen den beiden $(8,0)$-Versuchsgruppen kann kein solcher Test durchgeführt werden. Im TakingSpiel $(8,0)$ würde das Abgeben von 0 Gummibärchen reziprokes und Nehmen eigennütziges Verhalten bedeuten. Im sequenziellen Diktatorspiel $(8,0)$ können die beiden Verhaltensweisen hingegen nicht unterschieden werden: Das ERC-Modell prognostiziert übereinstimmend mit klassischen Ansätzen, dass sämtliche Individuen nichts abgeben.

8 Vor dem Versuch wurde das Einverständnis der Eltern eingeholt. Es kam zu keinen Verweigerungen.

9 Die Instruktionen zu den 4 Versuchsbedingungen finden sich im Anhang. Der genaue Wortlaut der allgemeinen Einführung zum Experiment kann auf Anfrage beim Autor bezogen werden: joel.berger@soz.unibe.ch. 
Schüler dieser Klasse ebenfalls 8 Gummibärchen und zusätzlich Informationen über die Entscheidung des anderen Kindes, des sogenannten ,Partnerkindes ${ }^{6} .{ }^{10}$ Jede Schülerin und jeder Schüler könne sich zuerst darüber informieren, wie das jeweils andere Kind seine Belohnung aufgeteilt hat und sich danach entscheiden, wie es die eigene aufteilen möchte.

Während die Klasse durch Einzelarbeit beschäftigt war, erhielt der Reihe nach ein Schulkind von der Lehrperson einen Umschlag und ging damit vor das Schulzimmer, wo es seine Belohnung aufteilte.

Probandinnen und Probanden der Versuchsgruppe $D(8,0)$ erhielten einen Umschlag mit 8 Gummibärchen. Die beigelegte Karte (vgl. Anhang) informierte darüber, dass das Partnerkind alle 8 Gummibärchen selber behalten hatte. Die Versuchsperson konnte sich nun entscheiden, wie viele seiner eigenen 8 Einheiten es dem anderen Kind abgeben wollte. Zu diesem Zweck konnte es auf der beigelegten Karte die entsprechende Kategorie ( 0 geben, 1 geben,..., 8 geben) ankreuzen. In der Versuchsgruppe $D(5,3)$ waren die Instruktionen identisch bis auf den Hinweis, dass sich das Partnerkind entschieden habe, 3 Gummibärchen abzugehen und sich daher 11 Stück im Umschlag befänden.

In der Versuchsgruppe $T(8,0)$ waren wiederum 8 Gummibärchen im Umschlag. Die Anweisungen entsprachen denjenigen im Diktatorspiel $(8,0)$, ausgenommen den Vermerk, dass nicht nur bis zu 8 Gummibärchen abgegeben, sondern alternativ bis zu 8 eingefordert werden können. Die Entscheidung erfolgte durch das Ankreuzen der entsprechenden Kategorie (8 nehmen, 7 nehmen,..., 0 nehmen, 1 geben, 2 geben,..., 8 geben). In der Versuchsgruppe T(5, 3) befanden sich 11 Belohnungseinheiten im Umschlag. Die Instruktionen unterschieden sich von denjenigen in $\mathrm{T}(8,0)$ durch die Information, dass sich das Partnerkind entschieden habe, 3 Stück abzugehen und sich darum 11 Einheiten im Umschlag befänden. Dementsprechend weicht die Skala von jener in der Versuchsbedingung $\mathrm{T}(8,0)$ ab: Die Versuchsperson konnte maximal 5 zusätzliche Einheiten nehmen, da sie bereits 3 Stück von Spieler 1 erhalten hat.

Nach der Entscheidung klebte die Probandin bzw. der Proband den Umschlag mit den Gummibärchen und der Karte zu und warf ihn in eine Urne. Aus den einführenden Informationen der Lehrperson und aufgrund eines Vermerks auf der Karte war bekannt, dass die Belohnung unmittelbar nach dem Experiment den Entscheidungen entsprechend aufgeteilt würde und dass die Entscheidungen völlig anonym blieben. ${ }^{11}$ Nachdem dem Aufteilen der Belohnung füllte jedes Kind einen kurzen Fragebogen aus. Dabei wurde das Verständnis der jeweiligen Experimentalbedingung geprüft und die Präferenz für Gummibärchen abgefragt. Zusätzlich wurde eine offene Frage nach der Motivation der Entscheidung gestellt. Der Fragebogen und die Karte wurden mit demselben Code versehen, damit eine gegenseitige Zuordnung der beiden Beobachtungen möglich ist.

\section{Empirische Analysen}

Vor der Durchführung der Analysen wurde getestet, ob die Fälle aus den einzelnen Schulklassen gepoolt werden können, da Beobachtungen innerhalb sozialer Einheiten nicht a priori als statistisch unabhängig betrachtet werden können (Maddala 2005: 228). Hierzu wurde die Anzahl der abgegebenen Belohnungseinheiten auf Dummy-Variablen für die einzelnen Schulklassen regressiert. Es konnten keine signifikanten Unterschiede zwischen den Klassen ge-

10 Der Begriff ,Partnerkind“ wurde gewählt, um klarzustellen, dass die Zweiergruppen nach der ersten Runde nicht neu gemischt werden.

11 Ist Anonymität bezüglich der Entscheidungen - und zwar sowohl zwischen den Versuchspersonen als auch zwischen den Versuchspersonen und dem Versuchsleiter - nicht gewährleistet, sind die Entscheidungen in Richtung sozialer Erwünschtheit verzerrt (Chaudhuri 1999, S. 57; Bohnet 2009, S. 651 f.). 
funden werden, die Daten können folglich für die Analysen zum Zusammenhang von Treatment-Gruppe und Abgabeverhalten gepoolt werden.

Mittels Fragen über die Aufteilung der Belohnung wurde kontrolliert, ob die Kinder die Anweisungen verstanden haben. Während für das Diktatorspiel keine Anhaltspunkte für Verständnisschwierigkeiten vorliegen, müssen beim Taking-Spiel 13 Personen aus den Analysen ausgeschlossen werden. ${ }^{12}$ Durch den Ausschluss der fehlerhaften Beobachtungen resultiert eine Nettostichprobe von $n=83$. Weiter wurden die Kinder gefragt, ob sie Gummibärchen nicht mögen, mittelmäßig mögen oder gern mögen. Von den 83 Kindern mögen 12 Kinder Gummibärchen nur mittelmäßig, alle anderen mögen sie gut; kein einziges Kind berichtete tiefe Präferenzen. ${ }^{13}$

\section{a) Vergleich der Diktator-Versuchsbedingungen}

Im sequenziellen Diktatorspiel (8, 0) erhalten die Spieler 2 von den (fiktiven) Spielern 1 keine Gummibärchen. 14 von 24 (58.3\%) der Spieler 2 gaben ebenfalls nichts ab. Der Median beträgt 0, der arithmetische Mittelwert 1.29. Diese Differenz ist auf die Schiefe der Verteilung zurückzuführen: Die zweithäufigste Kategorie ist 4, eine Person gibt 7 Einheiten ab. Dass die Verteilung freundlicher ausfällt als theoretisch erwartet, könnte damit zu tun haben, dass Kinder beim Aufteilen von Süßigkeiten großzügiger vorgehen als beim Aufteilen von Geld (Murningham / Saxon 1998, S. 439). Auch soziale Erwünschtheit könnte eine Rolle spielen. Allerdings dürften aufgrund der Randomisierung dieselben Effekte im sequenziellen Diktatorspiel $(5,3)$ wirken, wo Spieler 2 jeweils 3 Gummibärchen als Vorleistung erhält. Die relative Mehrheit der Spieler 2 (9 von 23; 39.1\%) reagiert auf diese Vorleistung, indem sie 4 Einheiten abgeben, nur 4 Kinder behalten alles für sich; 82.6\% geben mindestens eine Einheit ab. Der Median beträgt 3, das arithmetische Mittel 2.61. Die zweithäufigsten Optionen sind 0 und 2.

12 Die betroffenen Fälle bezogen die Optionen ,Nehmen` und ,Geben' auf die 8 bzw. 11 Einheiten in ihrem Umschlag und führten gewissermaßen ein Diktatorspiel durch, indem sie jeweils zwei Kreuze anbrachten. Solche Fälle traten sowohl in der T $(8,0)$ - als auch in der T $(5,3)$-Versuchsgruppe auf und fügen den Daten ,Noise“ hinzu. Unter der Berücksichtigung solcher Fälle wird der Unterschied in der zentralen Tendenz weniger deutlich, verschwindet jedoch nicht ganz (robuste Verwahren wie die Median-Regression weisen die Differenz weiterhin nach, während ein Mittelwertvergleich keinen signifikanten Unterschied mehr anzeigt).

13 Sämtliche Analysen wurden inklusive dieser 12 Fälle durchgeführt. Werden sie ausgeschlossen, ändern sich die Resultate nicht signifikant. 
Abbildung 1: Sequenzielle Diktatorspiele

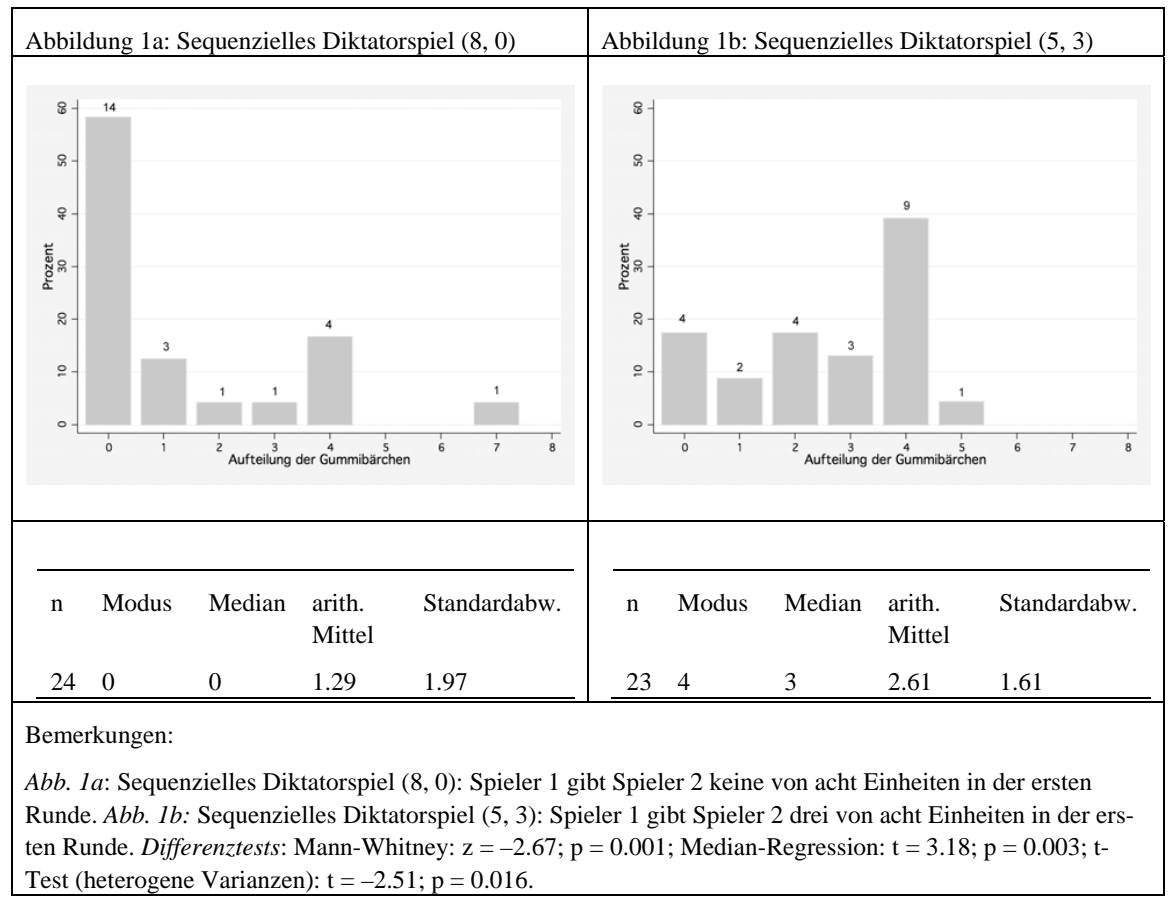

Der theoretisch erwartete Unterschied in der zentralen Tendenz zeigt sich sowohl beim Vergleich der Modi (0 vs. 4), der Mediane (0 vs. 3) als auch der arithmetischen Mittelwerte (1.29 vs. 2.61) der beiden Verteilungen. Die Differenzen erweisen sich sowohl im nonparametrischen Mann-Whitney Rangsummentest $(z=-2.67 ; p=0.001)$, in einer Median-Regression $(\mathrm{t}=3.18 ; \mathrm{p}=0.003)$, als auch einem $\mathrm{t}$-Test $(\mathrm{t}=-2.51 ; \mathrm{p}=0.016)$ als statistisch signifikant. ${ }^{14}$ Nicht mit der Modellvorhersage überein stimmt nur der Befund, dass die relative Mehrheit der Personen im Diktatorspiel mit der Auszahlung (5, 3) 4 von 8 Einheiten abgibt, obwohl die Vorleistung nur 3 beträgt.

\section{b) Vergleich der Taking-Versuchsbedingungen}

Das Taking-Spiel erlaubt es im Gegensatz zum Diktatorspiel, dem anderen Spieler Einheiten zu nehmen. Im sequenziellen Taking-Spiel $(8,0)$ erhalten die Spieler 2 von den (fiktiven) ersten Spielern 0 von maximal 8 Belohnungseinheiten. Die relative Mehrheit der zweiten Spieler, 6 von 17 Versuchspersonen (35.3\%), wählt ebenfalls die Null-Option. 5 Kinder nehmen Gummibärchen und 6 geben welche ab. Insgesamt entscheiden sich 70.5\% der Probandinnen und Probanden dafür, nichts zu geben oder zu nehmen - mehr als im Diktatorspiel. Offenbar hat die Skala einen gewissen Einfluss auf das Abgabeverhalten. Die Entscheidungen stimmen

14 Sämtliche berichteten Signifikanztests wurden zweiseitig durchgeführt. 
jedoch mit den Prognosen des ERC-Modells überein: Eigennützige Individuen nehmen und ungleichheitsaverse geben nichts ab.

Abbildung 2:

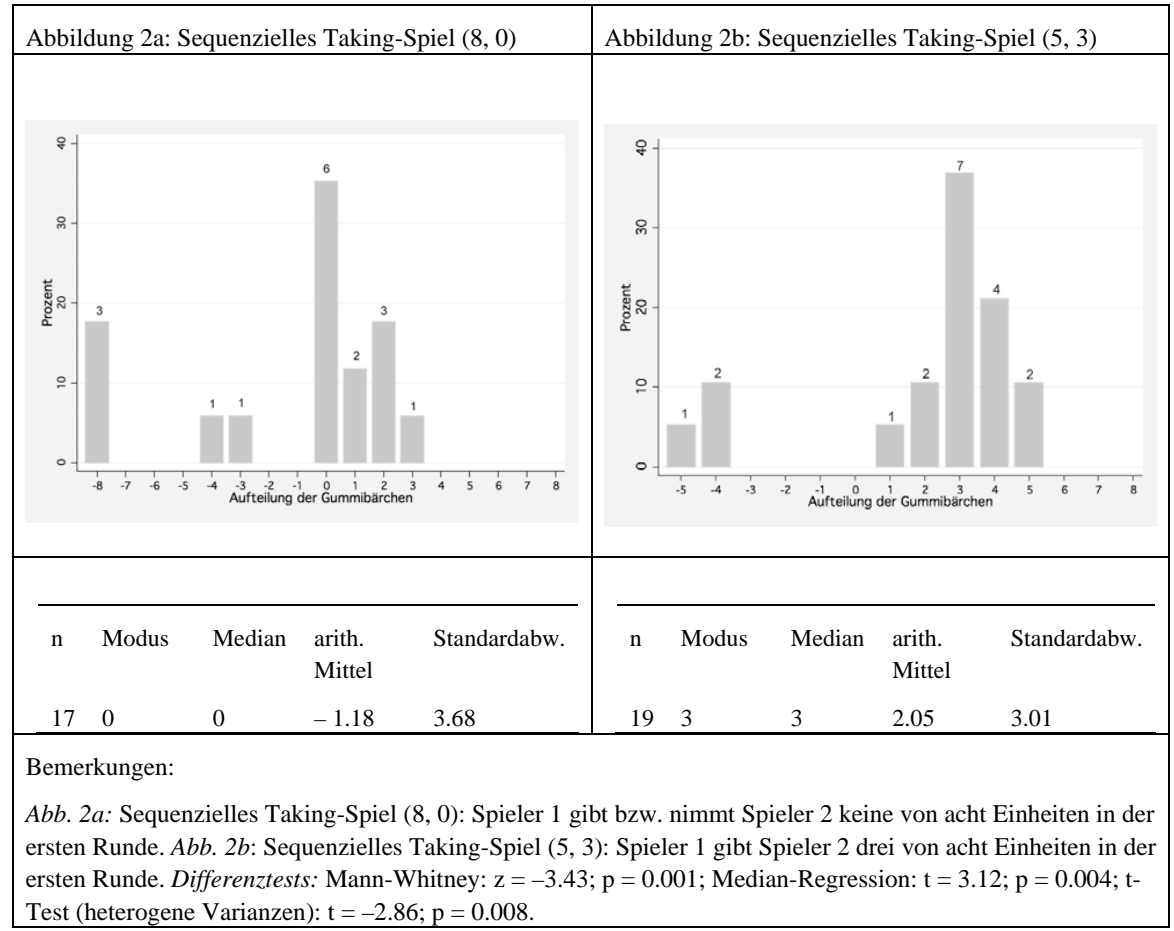

Erhalten im sequenziellen Taking-Spiel $(5,3)$ die zweiten von den ersten Spielern 3 Einheiten, gibt die relative Mehrheit (7 von 19;36.8\%) ebenfalls 3 zurück. Lediglich 3 Personen $(15.8 \%)$ nehmen Einheiten, die Option 0 wird nie gewählt. Die Verteilung unterscheidet sich trotz der eher geringen Fallzahl bezüglich der Mediane $(\mathrm{t}=3.12 ; \mathrm{p}=0.004)$ und der arithmetischen Mittelwerte deutlich $(\mathrm{t}=-2.86 ; \mathrm{p}=0.008)$. Eine Differenz in den zentralen Tendenzen kann auch mit dem Mann-Whitney Rangsummentest nachgewiesen werden $(\mathrm{z}=-3.43 ; \mathrm{p}=$ 0.001). ${ }^{15}$ Zudem zeigt sich ein weiterer Kategorieneffekt: Werden Vorleistungen im sequenziellen Diktatorspiel mit 4 Belohnungseinheiten honoriert, sind es im Taking-Spiel nur noch drei. Offenbar lässt die zusätzliche Taking-Option die Abgabe von drei Einheiten als genügend freundlich erscheinen.

15 Es muss beachtet werden, dass die Skalen dieser beiden Bedingungen nicht dieselben sind: In der Bedingung $T(8,0)$ können bis zu 8 zusätzliche Gummibärchen genommen werden; in der Kondition $\mathrm{T}(5,3)$ maximal 5 , da der erste Spieler bereits 3 an den zweiten Spieler abgegeben hat. Es ist jedoch nicht anzunehmen, dass dieser Umstand die Differenz in der zentralen Tendenz der beiden Verteilungen erklärt. Einerseits liegen beide Modi im positiven Bereich der jeweiligen Skala. Andererseits liegen nur wenige Beobachtungen im negativen Bereich. Insbesondere die robusten Tests dürften daher kaum verzerrt worden sein. 


\section{c) Vergleich der (5, 3)-Versuchsbedingungen}

Auch wenn die Orientierung an einem normativen Bezugspunkt beim Vorliegen einer TakingOption nicht erodiert, ist es prinzipiell möglich, dass sich im sequenziellen Taking-Spiel (5, 3) weniger Individuen an einer solchen Norm orientieren als im sequenziellen Diktatorspiel $(5,3)$. Nachdem mit der Einführung der Taking-Option das reziproke Verhalten infolge der erhöhten Auszahlung bei Ausbeutung und einer Verschiebung des Skalenmitte in Richtung Eigennutz einem härteren Test unterzogen wurde, wird abschließend untersucht, wie robust normorientiertes Verhalten im Verhältnis zu verzerrenden Effekten ist. Wenn die Annahmen des ERC-Modells zutreffen und das Abgeben von Einheiten durch die Orientierung an einem normativen Bezugspunkt motiviert ist, dürfte die Taking-Option nicht dazu führen, dass der Anteil an Personen, welche die Vorleistung von Spieler 1 mit fairem Verhalten erwidert, in der Taking-Versuchsgruppe $(5,3)$ geringer ist als in der Diktator-Versuchsgruppe $(5,3)$. Das ERC-Modell lässt jedoch zu, dass sich Personen eigennützig verhalten. Somit ist zu erwarten, dass Akteure mit stark eigennützigen Präferenzen die Taking-Option nutzen, wodurch sich die beiden Versuchsgruppen in ihrer zentralen Tendenz durchaus unterscheiden dürfen. Als reziprok wird für beide Versuchsbedingungen dass Abgeben von mehr als null Belohnungseinheiten definiert, eigennütziges Verhalten ist das Behalten sämtlicher Einheiten und in der Versuchsgruppe $\mathrm{T}(5,3)$ zusätzlich das Nehmen von Gummibärchen. ${ }^{16}$

Im sequenziellen Diktatorspiel (5, 3) verhalten sich 4 von 23 Akteuren (17.4\%) eigennützig, 19 (82.6\%) honorieren die Vorleistung des ersten Spielers. Im sequenziellen Taking-Spiel (5, 3) nutzen 3 von 19 (15.8\%) der Versuchspersonen die Taking-Option, 16 von 19 (84.2\%) geben mindestens eine Einheit ab; die Option 0 wird nie gewählt. Die Differenz der beiden Verteilungen ist mit $\mathrm{ml}^{2}=0.02(\mathrm{p}=0.890)$ deutlich insignifikant. ${ }^{17}$ Der relative Anteil an Personen, deren Entscheidung nicht nur durch Eigennutz, sondern auch durch eine Reziprozitätsnorm beeinflusst wird, unterscheidet sich zwischen dem Taking- und dem Diktatorspiel nicht. Dieses Resultat ist ein weiterer Hinweis für die Gültigkeit des ERC-Modells.

\section{Diskussion}

Sowohl im sequenziellen Diktator- als auch im sequenziellen Taking-Spiel wurde die Vorleistung von 3 Einheiten des ersten Spielers erwidert. Während im Diktatorspiel $(5,3)$ der Modalwert des zweiten Spielers 4 Belohnungseinheiten beträgt, gibt die relative Mehrheit im Taking-Spiel 3 zurück. Beide Resultate sind ein deutlicher Hinweis für reziprokes Verhalten von 11-jährigen Kindern und stimmen prinzipiell mit den Befunden von Diekmann (2004) und Ben-Ner et al. (2004) überein. Weitere Analysen legen offen, dass der Anteil der Akteure, der sich nicht rein eigennützig verhält, sich zwischen der Diktator-Versuchsbedingung $(5,3)$ und der Taking-Bedingung $(5,3)$ nicht unterscheidet. Dieser Befund legt nahe, dass durchaus eine Orientierung der Entscheidungen an einem als fair erachteten Punkt - und nicht die Orientierung an den zur Verfügung stehenden Optionen - die Entscheidungen substantiell beeinflusst hat.

Das ERC-Modell prognostiziert in der Versuchsbedingung $\mathrm{D}(5,3)$ eine Verteilung von 0 bis 3 Belohnungseinheiten: Gibt der zweite Spieler 3 Einheiten an Spieler 1 zurück, verfügt er über 8 von 16 Gummibärchen. In der Bedingung T $(5,3)$ wäre eine Verteilung von -5 bis $3 \mathrm{zu}$

16 Eine Alternative wäre, das Nehmen von weniger als 5 Gummibärchen auch als von Fairnessüberlegungen beeinflusste Entscheidung zu betrachten und nur das Nehmen von 5 Einheiten als eigennütziges Verhalten zu definieren. Durch die hier verfolgte Definition werden die eigennützigen Verhaltensweisen im Vergleich zu fairen eher gestärkt, was einem härteren Test der Reziprozitäts-Interpretation gleichkommt.

17 Einseitiger Test 
erwarten. Überraschend entscheidet sich im sequenziellen Diktatorspiel die relative Mehrheit (9 von 23) dafür, 4 Stück an Spieler 1 abzugeben. Solche Resultate beobachten Ben-Ner et al. (2004) und Diekmann (2004) bei einer Studierenden-Stichprobe nicht. Bei letzterer Studie erwidert Spieler 2 eine Vorleistung von 20\% des Betrags ebenfalls mit 20\%; als Reaktion auf 5 werden 5 Einheiten zurückgegeben und 6 werden mit 6 honoriert (Diekmann 2004: 498).

Eine Ad-hoc-Erklärung für die unterschiedlichen Resultate wäre, dass es prinzipiell zwei als fair erachtete Punkte gibt - nämlich einerseits die eigene Belohnung in zwei gleich große Hälften zu teilen und andererseits die Handlung von Spieler 1 exakt zu spiegeln. Nun ist ein Gummibärchen nicht sehr kostspielig - so berichten Muringham / Saxon (1998), dass Kinder mit Süßigkeiten im Vergleich zu Geld eher großzügig umgehen. Es ist anzunehmen, dass sich angesichts der geringen Kosten eines Gummibärchens im Diktatorspiel (5,3) die freundlichere Variante anbietet, während im Taking-Spiel der weniger nette Bezugspunkt,drei zurückgeben angesichts der Taking-Option genug freundlich erscheint. Es könnte somit ein Kategorieneffekt vorliegen.

Viele Antworten auf die Frage nach der verwendeten Entscheidungsregel lassen darauf schließen, dass die meisten Schülerinnen und Schüler gerade in der T(5, 3)-Bedingung ihr Verhalten eng an einer Reziprozitätsnorm orientiert haben: „Mein(e) Partner(in) hat mir 3 gegeben und ich habe ihr 3 gegeben. Weil es dann fair ist." In der $\mathrm{D}(5,3)$-Bedingung überwiegen hingegen Aussagen der folgenden Art: „Ich habe ihm 4 gegeben weil ich ihm nicht gleichviel sondern 1 mehr geben wollte. "Die Antworten der Kinder stützten also die Ad-hocInterpretation.

Ad-hoc-Deutungen liefern zwar keine Antworten, jedoch Hinweise dafür, in welche Richtung sinnvoll weiter geforscht werden kann. Erstens könnten der Gesamtbetrag und die Gabe von Spieler 1 so variiert werden, dass die beiden Bezugspunkte, nämlich einerseits die eigene Belohnung fair aufzuteilen und andererseits alle Gummibärchen fair aufzuteilen, weiter voneinander entfernt liegen. Zweitens wäre es interessant, Geld als Incentive einzusetzen um zu klären, ob damit tatsächlich weniger großzügig umgegangen wird. ${ }^{18}$ Drittens wäre es vielversprechend, die Entwicklung der Reziprozität bei Kindern verschiedener Altersgruppen zu beobachten. Werden solche Beobachtungen mit Daten zu den individuellen Sozialisationsbedingungen kombiniert, können Hinweise darüber gewonnen werden, inwiefern die Internalisierung einer Reziprozitätsnorm durch die Qualität der sozialisatorischen Umgebung beeinflusst wird (vgl. Benenson / Pascoe / Radmore 2007: 171 f).

Im Fazit kann festgehalten werden, dass altruistische Reziprozität bereits bei 11-jährigen Grundschulkindern sowohl im sequenziellen Diktator- als auch im sequenziellen Taking-Spiel nachgewiesen werden kann. Offenbar orientieren bereits Kinder, die ihren Sozialisationsprozess noch nicht vollendet haben, ihr Tauschverhalten an einer Reziprozitätsnorm. Diese Resultate stärken Alvin Gouldners Argumentation: Neben strategisch motivierter Reziprozität scheint eine internalisierte Reziprozitätsnorm zu existieren, welche einen Kontrapunkt zum reinen Eigeninteresse darstellt und dadurch zur Stabilität sozialer Systeme beiträgt.

\section{Literatur}

Axelrod, R. (1984): The Evolution of Cooperation, New York.

Bardsley, N. (2008): Dictator Game Giving: Altruism or Artefact?, in: Experimental Economics 11, S. 122-133.

18 In der zitierten Studie von Murningham / Saxon (1998) wurden keine reellen Incentives eingesetzt, sondern Fragen danach gestellt, wie sich die Kinder in bestimmten Situationen entscheiden würden (vgl. hierzu Camerer 2006: 38ff). 
Bardsley, N. / R. Cubitt / G. Loomes / P. Moffatt / C. Starmer / R. Sugden (2010): Experimental Economics. Rethinking the Rules, Princeton.

Batson D. C. / T. Moran (1999): Empathy-Induced Altruism in a Prisoner's Dilemma, in: European Journal of Social Psychology 29, S. 909-924.

Benenson, J. F. / Pascoe, J. / Radmore, N. (2007): Children's Altruistic Behavior in the Dictator Game, in: Evolution and Human Behavior 28, S. 168-175.

Ben-Ner, A. / L. Putterman / F. Kong / D. Magan (2004): Reciprocity in a Two-Part Dictator Game. Journal of Economic Behavoir \& Organization 53, S. 333-352.

Bohnet, I. (2009): Experiments, in: P. Hedström / P. Bearman: The Oxford Handbook of Analytical Sociology, Oxford, S. 639-665.

Bolton, G. E. / A. Ockenfels (2000): A Theory of Equity, Reciprocity, and Competition, in: The Amercian Economic Review, S. 166-193.

Camerer, C. F. (2006): Behavioral Game Theory, New York.

Chaudhuri, A. (2009): Experiments in Economics. Playing Fair with Money, London.

Damon, W. (1984): Die soziale Welt des Kindes, Frankfurt a. M.

Diekmann, A. (2004): The Power of Reciprocity. Fairness, Reciprocity, and Stakes in Variants of the Dictator Game, in: Journal of Conflict Resolution 487, S. 487-505.

Diekmann, A. (2009): Spieltheorie. Einführung, Beispiele, Experimente, Reinbek bei Hamburg.

Diekmann, A. / D. Wyder (2002): Vertrauen und Reputationseffekte bei Internet-Auktionen, in: Kölner Zeitschrift für Soziologie und Sozialpsychologie 54, S. 674-693.

Falk, A. / E. Fehr / U. Fischbacher (2008): Testing Theories of Fairness - Intentions Matter, in: Games and Economic Behavior 62, S. 287-303.

Fehr, E. / H. Bernhard / B. Rockenbach (2008): Egalitarianism in Young Children, in: Nature 454, S. 1079-1084.

Fehr, E. / Fischbacher, U. (2003): The Nature of Human Altruism. Nature 425, S. 785-791.

Fehr, E. / Fischbacher, U. (2004): Third-Party Punishment and Social Norms, in: Evolution and Human Behavior, 25, S. 63-87.

Fehr, E. / H. Gintis (2007): Human Motivation and Social Cooperation: Experimental and Analytical Fundations, in: Annual Review of Sociology 33, S. 43-64.

Fehr, E. / G. Kirchsteiger / A. Riedl (1993): Does Fairness Prevent Market Clearing? An Experimental Investigation, in: The Quarterly Journal of Economics 108, S. 437-459.

Gouldner, A. W. (1960): The Norm of Reciprocity. A Preliminary Statement, in: American Sociological Review 25, S. 161-178.

Henrich, J. / R. McElreath / A. Barr / J. Ensminger / C. Barrett et al. (2006): Costly Punishment Across Human Societies, in: Science 312, S. 1767-1770.

Hobbes T. (1968): Leviathan, Harmondsworth.

Kienbaum, J. / B. Schuhrke (2010): Entwicklungspsychologie der Kindheit. Von der Geburt bis zum 12. Lebensjahr. Grundriss der Psychologie, Band 13, Stuttgart.

Kienbaum, J. / F. Wilkening (2009): Children's and Adolescent's Intuitive Judgment about Distributive Justice: Integrating Need, Effort, and Luck, in: European Journal of Developmental Psychology 6, S. 481-498.

List, J. A. (2007): On the Interpretation of Giving in Dictator Games, in: Journal of Political Economy 115 , S. 482-493.

Maddala, G. S. (2005): Introduction to Econometrics, Chichester.

Malinowski, B. (1926): Crime and Custom in Savage Society, London. 
Murningham, K. J. / Michael S. Saxon (1998): Ultimatum Bargaining by Children and Adults, in: Journal of Economic Psychology 19, S. 415-445.

Nowak, M. A. / K. Sigmund (2005): Evolution of Indirect Reciprocity, in: Nature 437, S. 1291-1298.

Ockenfels, A. (1999): Fairness, Reziprozität und Eigennutz, Tübingen.

Orne, M. T. (1962): On the Social Psychology of the Psychological Experiment: With Particular Reference to Demand Characteristics and their Implications. Amercian Psychologist 17, S. 776-783.

Parducci, A. / D. H. Wedell (1986): The Category Effect With Rating Scales: Number of Categories, Number of Stimuli, and Method of Presentation. Journal of Experimental Psychology: Human Perception and Performance 12, 496-516.

Parsons, T. (1937): The Structure of Social Action, New York.

Premack, D. / G. Woodruff (1978): Does the Chimpanzee Have a Theory of Mind?, in: Behavioral and Brain Sciences 4, S. 515-526.

Robinson, J. L. (2008): Empathy and Prosocial Behavior, in: R. V. Kail (Hrsg.): Children and their Development, Upper Saddle River, S. 441-450.

Simmel, G. (1950): The Sociology of Georg Simmel, Glencoe.

Takagishi, H. / S. Kameshima / J. Schug / M. Koizumi / T. Yamagishi (2010): Theory of Mind Enhances Preference for Fairness, in: Journal of Experimental Child Psychology 105, S. 130-137.

Willer, R. (2009): Groups Reward Individual Sacrifice: The Status Solution to the Collective Action Problem, in: American Sociological Review 74, S. 23-43.

Joël Berger Universität Bern Institut für Soziologie

Lerchenweg 36

CH-3000 Bern 9

joel.berger@soz.unibe.ch 


\section{Anhang}

a) Instruktionen zur Versuchsbedingung $D(8,0)$

Lies bitte diesen Text gut durch. Wenn du nicht draus kommst, dann Frage deine Lehrperson.

Jedes Kind bekommt 8 Gummibärchen, weil es bei diesem Versuch mitmacht. Deine 8 Gummibärchen sind in diesem Umschlag. Dein Partnerkind hat auch 8 Gummibärchen bekommen.

Dein Partnerkind konnte sich entscheiden, ob es dir von seinen 8 Gummibärchen einige Gummibärchen geben wollte. Es hat sich dazu entschieden, dir keine Gummibärchen zu geben von seinen 8 Gummibärchen.

\section{Nun musst du dich entscheiden:}

- Du darfst dem Partnerkind so viele von deinen 8 Gummibärchen geben, wie du willst.

- Du musst dem Partnerkind keine Gummibärchen geben, wenn du nicht willst.

Kreuze an, was du tun möchtest:

$\begin{array}{lllllllll}\square & \square & \square & \square & \square & \square & \square & \square & \square \\ 0 & 1 & 2 & 3 & 4 & 5 & 6 & 7 & 8 \\ \text { geben } & \text { geben } & \text { geben } & \text { geben } & \text { geben } & \text { geben } & \text { geben } & \text { geben } & \text { geben }\end{array}$

Tue nun dieses Blatt Papier und die Gummibärchen in den Umschlag und klebe den Umschlag zu. Nach dem Versuch kriegen alle Kinder die Gummibärchen, die ihnen gehören. Auch du kriegst noch heute deine Gummibärchen.

\section{b) Instruktionen zur Versuchsbedingung $D(5,3)$}

Lies bitte diesen Text gut durch. Wenn du nicht draus kommst, dann Frage deine Lehrperson.

Jedes Kind bekommt 8 Gummibärchen, weil es bei diesem Versuch mitmacht. Deine 8 Gummibärchen sind in diesem Umschlag. Dein Partnerkind hat auch 8 Gummibärchen bekommen.

Dein Partnerkind konnte sich entscheiden, ob es dir von seinen 8 Gummibärchen einige Gummibärchen geben wollte. Es hat sich dazu entschieden, dir 3 Gummibärchen zu geben von seinen 8 Gummibärchen. Darum hat es in deinem Couvert jetzt $8+3=11$ Gummibärchen. Sie gehören alle dir.

\section{Nun musst du dich entscheiden:}

- Du darfst dem Partnerkind so viele von deinen ursprünglichen 8 Gummibärchen geben, wie du willst.

- Du musst dem Partnerkind keine Gummibärchen geben, wenn du nicht willst.

Kreuze an, was du tun möchtest:

$\begin{array}{lllllllll}\square & \square & \square & \square & \square & \square & \square & \square & \square \\ 0 & 1 & 2 & 3 & 4 & 5 & 6 & 7 & 8 \\ \text { geben } & \text { geben } & \text { geben } & \text { geben } & \text { geben } & \text { geben } & \text { geben } & \text { geben } & \text { geben }\end{array}$


Tue nun dieses Blatt Papier und die Gummibärchen in den Umschlag und klebe den Umschlag zu. Nach dem Versuch kriegen alle Kinder die Gummibärchen, die ihnen gehören. Auch du kriegst noch heute deine Gummibärchen.

\section{c) Instruktionen zur Versuchsbedingung $T(8,0)$}

Lies bitte diesen Text gut durch. Wenn du nicht draus kommst, dann Frage deine Lehrperson.

Jedes Kind bekommt 8 Gummibärchen, weil es bei diesem Versuch mitmacht. Deine 8 Gummibärchen sind in diesem Umschlag. Dein Partnerkind hat auch 8 Gummibärchen bekommen.

Dein Partnerkind konnte sich entscheiden,

- ob es dir von seinen 8 Gummibärchen einige Gummibärchen geben wollte

- oder ob es von dir einige Gummibärchen wegnehmen wollte

- oder ob es nichts von beidem tun wollte.

Das Partnerkind hat sich dazu entschieden, dir keine Gummibärchen zu geben von seinen 8 Gummibärchen. Es wollte dir auch keine von deinen Gummibärchen wegnehmen.

\section{Nun musst du dich entscheiden:}

- Du kannst dem Partnerkind so viele von deinen 8 Gummibärchen geben, wie du willst.

- Du musst dem Partnerkind keine Gummibärchen geben, wenn du nicht willst.

- Du kannst vom Partnerkind so viele von seinen Gummibärchen nehmen, wie du willst. Kreuze an, was du tun möchtest:

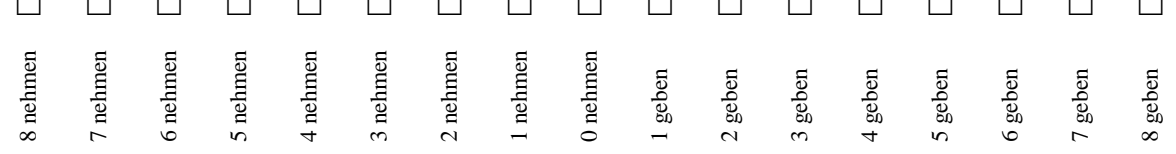

Tue nun dieses Blatt Papier und die Gummibärchen in den Umschlag und klebe den Umschlag zu. Nach dem Versuch kriegen alle Kinder die Gummibärchen, die ihnen gehören. Auch du kriegst noch heute deine Gummibärchen.

\section{d) Instruktionen zur Versuchsbedingung $T(5,3)$}

Lies bitte diesen Text gut durch. Wenn du nicht draus kommst, dann Frage deine Lehrperson.

Jedes Kind bekommt 8 Gummibärchen, weil es bei diesem Versuch mitmacht. Deine 8 Gummibärchen sind in diesem Umschlag.

Dein Partnerkind konnte sich entscheiden,

- ob es dir von seinen 8 Gummibärchen einige Gummibärchen geben wollte

- oder ob es von dir einige Gummibärchen wegnehmen wollte

- oder ob es nichts von beidem tun wollte.

Das Partnerkind hat sich dazu entschieden, dir 3 Gummibärchen zu geben von seinen 8 Gummibärchen. Darum hat es in deinem Couvert jetzt $8+3=11$ Gummibärchen. Sie gehören alle dir. 


\section{Nun musst du dich entscheiden:}

- Du darfst dem Partnerkind nun so viele von deinen ursprünglichen 8 Gummibärchen geben, wie du willst.

- Du musst dem Partnerkind keine Gummibärchen geben, wenn du nicht willst.

- Du kannst vom Partnerkind so viele von seinen Gummibärchen nehmen, wie du willst. Kreuze an, was du tun möchtest:

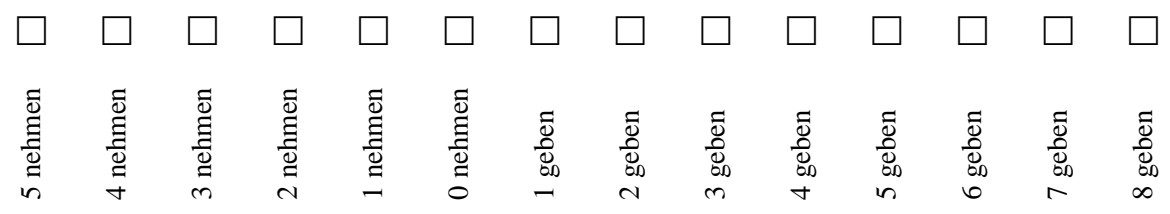

Tue nun dieses Blatt Papier und die Gummibärchen in den Umschlag und klebe den Umschlag zu. Nach dem Versuch kriegen alle Kinder die Gummibärchen, die ihnen gehören. Auch du kriegst noch heute deine Gummibärchen. 